\title{
ON THE APPLICATION OF HYBRID BEM/FEM FOR INHOMOGENEOUS ELECTROMAGNETIC SCATTERING FROM SMALL MARITIME TARGETS
}

\author{
HRVOJE DODIG ${ }^{1}$, DRAGAN POLJAK ${ }^{2} \&$ MARIO CVETKOVIĆ $^{2}$ \\ ${ }^{1}$ Faculty of Maritime Studies, University of Split, Croatia \\ ${ }^{2}$ Faculty of Electrical Engineering, Mechanical Engineering and Naval Architecture, University of Split, Croatia
}

\begin{abstract}
From the standpoint of detectability of small maritime objects by a ship's radar system, the radar cross section (RCS) of a maritime object is a key parameter that determines the detectability of an object. When a vessel navigates in narrow channels and in areas with high sea traffic density, the ability to detect small maritime targets such as buoys and inflatable rubber boats is crucial for maritime safety. However, it is difficult to measure the radar cross section of such maritime objects in environments such as laboratory or in a field test not only due to object size, but also due to the fact that these objects are partially submerged into seawater. In such situations, the most effective approach is to apply numerical methods for electromagnetic scattering from inhomogeneous objects in order to predict the RCS of a maritime object. Though approaches to RCS prediction are diverse, one of the most sophisticated, namely, the hybrid numerical method based on combination of boundary and finite elements is applied in this work. This hybrid numerical method first computes near electromagnetic field on the surface of the scatterer, followed by a particular technique developed by the authors to compute the radar cross section of small maritime targets from these near field values. The accuracy of the approach is discussed and some illustrative computational examples of RCS calculations are given. Keywords: boundary element method, finite element methods, hybrid boundary element/finite element method, electromagnetic scattering, radar cross section, small maritime targets.
\end{abstract}

\section{INTRODUCTION}

In coastal waters near tourist areas where dense maritime traffic is expected, the ability to detect small vessels such as inflatable rubber boats and buoys is of high importance. During night cruises or in bad weather conditions, the only sensor available to pilots and captains that can detect such objects is radar. The detection probability of these small maritime targets is directly proportional to an electrical engineering parameter called radar cross section (RCS). The radar cross section represents the susceptibility of radar target to detection by a radar [1], and it is the parameter that represents the ratio of backscattered power to transmitted power. Consequently, the lower the RCS, the smaller is probability of detection of radar target by a ship's radar.

While RCS does not necessarily depend on target's physical size, the most important factors are target's geometry and material composition. For example, radar-deflectors, sometimes called radar target enhancers (RTE), reflect radar antenna energy in such a way that the target appears larger on radar's display [2]. RTEs are physically small in size and their typical physical dimensions vary from $8 \mathrm{~cm}$ to $20 \mathrm{~cm}$. These devices are made of metal, and their geometry is designed in such a way that it enhances the RCS. On the other hand, inflatable rubber boats typically consist of materials such as plastic, wood and rubber which contribute very little to RCS. This impairs the detection probability of rubber boat by ships radar, thus, many of these inflatable rubber boats install radar reflectors to improve the navigational safety and to decrease the chance of collision with larger vessel.

In the design of radar systems with the ability to detect these small maritime objects it is advantageous to know in advance the RCS to be expected. It is very difficult to realistically 
measure RCS of rubber boats and buoys in laboratory conditions because of the sheer size of these objects and because these objects are partially submerged into seawater. On the other hand, if field measurements are performed, the difficulties in RCS measurement arise from not being able to control weather conditions and the sea state such as waves and currents. Hence, perhaps the most cost effective and reliable solution to RCS prediction is to numerically calculate RCS of these small maritime objects.

Numerical methods that can be applied in order to predict the RCS of small maritime target generally fall into one of the following categories: ray-tracing methods [3], [4], raytracing combined with physical optics methods (PO) or with physical theory of diffraction (PTD) [5], [6], and full wave methods based on hybrid method of moments/finite element method (MoM/FEM) or hybrid boundary element method/finite element method (BEM/FEM) [7]. Because of multiple reflections of rays between facets [8], ray tracing methods are sometimes difficult to apply if the geometry of the target has highly irregular features. Raytracing combined with PO or PTD can produce RCS calculation with acceptable error for homogeneous scatterers, however, these methods can have difficulties when there are material inhomogeneities within the penetration depth of radar wave.

Full wave methods that can be used for RCS prediction are FDTD [9], hybrid MoM/FEM [10], volume-surface integral equation (VSIE) and hybrid BEM/FEM [7]. These methods come with the cost of increased computational burden when compared to raytracing with PO/PTD methods. However, they can properly take into account inhomogeneities of target's material composition. FDTD can sometimes have problems with irregular surfaces because of staircasing. VSIE methods combine surface integral equation with volume integral equation and MoM is typically used to assemble the linear system of equations. Consequently VSIE produces dense matrices for both surface and interior of the computational problem it is impractical for RCS computation of anything but the smallest objects.

Hybrid MoM/FEM method combines surface integral equation with FEM and it uses MoM to create a linear system of equations for the surface of electromagnetic scattering problem. Hence, MoM/FEM generates the linear system of equations which is sparse in the interior of the computational problem (because of FEM) and dense at the bounding surface of the computational problem (because of MoM). In the hybrid MoM/FEM method, typical elements used for surface discretization of an EM scattering problem are Rao-WiltonGlisson (RWG) elements. This can sometimes pose a difficulty because these elements are not divergenceless which is physically unrealistic; however, that method approaches nonzero divergence condition with increased surface discretization.

Thus, in order to implement a physically correct method for EM scattering computation which can take inhomogeneities of materials into account, in this paper we use the hybrid $\mathrm{BEM} / \mathrm{FEM}$ method with edge elements. Edge elements are used in hybrid BEM/FEM for electromagnetic scattering to model both interior of the problem (FEM part) and the surface of the problem (BEM part) which is an advantage in itself because the same type of elements is used to model interior and the surface of EM scattering problem. Furthermore, this type of elements bear two advantages: (i) edge elements are divergenceless and (ii) they automatically preserve tangential continuity of electromagnetic fields. Tangential continuity of electromagnetic fields is a physically required condition on the surfaces that are interface between materials with different electrical properties. Additional advantage of edge elements is that all the surface integrals in EM scattering computation with hybrid BEM/FEM that come from BEM can be converted to contour integrals [11]. Controlling the accuracy of the calculation of BEM integrals is significantly simpler when these integrals are computed as contour integrals instead of surface integrals. Finally, regarding hybrid BEM/FEM with edge elements, it should be noted that the research on this type of elements used in hybrid 
BEM/FEM started a long time ago, however, due to some mathematical difficulties the method did not became main-stream method compared to BEM/FEM. The resolution to some of these difficulties is reported in [12].

Regardless of the applied full wave method, the final result of numerical EM scattering computation is near electromagnetic field. This near electrical field needs to be converted to far field in order to compute the RCS. Standard near-to-far field transformation (NTFFT) is a computationally cumbersome procedure [13]. To circumvent this, the approach based on [14] is applied to compute the far field and RCS. The method is described in Section 3, and it allows rapid computation of RCS directly from edge element coefficients provided by near field computation.

In Section 4, the solid models of buoy and rubber boat, respectively, are given. While the buoy model was taken from technical specification of the buoy, the model of rubber boat is constructed by 3D laser scanning (courtesy of Croatian Navy). Finally, the results of numerical computation of the RCS of the buoy and the rubber boat are presented in Section 5 for both horizontal polarization (HP) and vertical polarization (VP) of EM wave. These results were compared to RCS of the sea itself in an attempt to predict the probability of detection.

\section{HYBRID BEM FEM FOR ELECTROMAGNETIC SCATTERING}

The first step in computation of RCS of radar target is to compute backscattered electric and magnetic field at the bounding surface $\partial \mathrm{V}$ of the computational problem shown in Fig. 1. If the surface $\partial \mathrm{V}$ is near the target then the computed electromagnetic field will be near electromagnetic field. In Fig. 1 the incident electromagnetic field denoted $\vec{E}_{i}$ and $\vec{H}_{i}$ excites

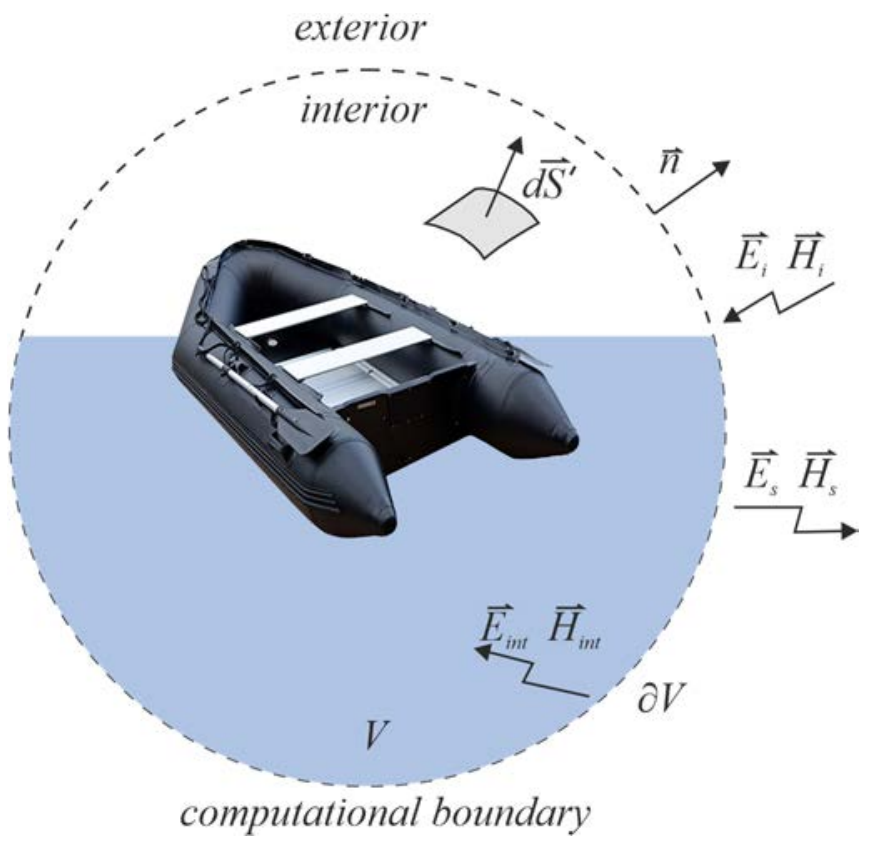

Figure 1: Outline of EM scattering problem. Volume of computational domain is denoted $V$ and the artificial boundary is denoted $\partial V$. Fields $\vec{E}_{i}$ and $\vec{H}_{i}$ are incident to $\partial V$ while $\vec{E}_{S}$ and $\vec{H}_{S}$ are backscattered fields. 
the sources on the surface of the scatterer which in turn generate backscattered electromagnetic field denoted $\vec{E}_{S}$ and $\vec{H}_{S}$. Fields interior to the computational domain are denoted $\vec{E}_{\text {int }}$ and $\vec{H}_{\text {int }}$. For the purpose of RCS computation we need to compute backscattered fields $\vec{E}_{S}$ and $\vec{H}_{S}$. However, the backscattered fields are affected by field distribution inside the scatterer and because the scatterer is considered to be inhomogeneous electromagnetic properties of materials $\left(\epsilon_{r}, \mu_{r}, \sigma\right)$ change inside computational domain $V$. Hence, to compute backscattered fields we need the computational method that can take into account this change of electromagnetic properties of materials.

The method that can properly take into account the changing electromagnetic properties of materials inside computational domain is hybrid BEM/FEM which is thoroughly described in references [12], [15], [16]. The starting point for hybrid BEM/FEM is magnetic field surface integral equation for time harmonic electromagnetic fields [17]:

$$
\begin{aligned}
\alpha \vec{H}_{e x t}= & \vec{H}_{i}+j \omega \epsilon \oint_{\partial V} d \vec{S}^{\prime} \times \vec{E}_{e x t}^{\prime} G+\oint_{\partial V}\left(d \vec{S}^{\prime} \times \vec{H}_{e x t}^{\prime}\right) \times \nabla^{\prime} G \\
& -\frac{j}{\omega \mu} \oint_{\partial V} d S^{\prime} \nabla_{S}^{\prime} \cdot\left(\vec{n}^{\prime} \times \vec{H}_{e x t}^{\prime}\right) \nabla^{\prime} G .
\end{aligned}
$$

In eqn (1), fields $\vec{E}_{\text {ext }}$ and $\vec{H}_{\text {ext }}$ denote the electric and magnetic fields exterior to the computational domain shown in Fig. 1. Fields interior to the computational domain (denoted $\vec{E}_{\text {int }}$ and $\vec{H}_{\text {int }}$ ) are governed by Maxwell equations. Two Maxwell equations that are necessary to implement FEM part of hybrid BEM/FEM method are Faraday's law and Maxwell-Ampere equation which can be written in time-harmonic form [18]:

$$
\begin{gathered}
\nabla^{\prime} \times \overrightarrow{\mathrm{E}}_{i n t}=-j \omega \mu \overrightarrow{\mathrm{H}}_{i n t}, \\
\nabla^{\prime} \times \overrightarrow{\mathrm{H}}_{i n t}=(\sigma+j \omega \epsilon) \overrightarrow{\mathrm{E}}_{i n t}
\end{gathered}
$$

Dividing eqn (2) by $-j \omega \mu$, and then taking the curl of eqn (2) and combining with eqn (3) yields:

$$
\nabla \times\left(\frac{\mathrm{j}}{\omega \mu} \nabla \times \overrightarrow{\mathrm{E}}_{\text {int }}\right)-(\sigma+j \omega \epsilon) \overrightarrow{\mathrm{H}}_{\text {int }}=0
$$

Let us now suppose that computational domain shown in Fig. 1 is discretized into nonconformal mesh consisting of tetrahedral elements and that fields $\vec{E}_{\text {int }}$ and $\vec{H}_{\text {int }}$ are approximated by some type of elements. The nodal elements cannot be used for this type of computation because these would produce spurious (nonphysical) results. However, one can use tangential edge elements [19] which physically correctly model the electromagnetic fields inside each tetrahedron. These type of elements are divergenceless which is physically required condition over each tetrahedron. Furthermore, edge elements preserve tangential continuity of electromagnetic fields across inter-element faces which is again physically required condition. Over each element, electric and magnetic fields can be approximated using edge elements as: 


$$
\begin{aligned}
& \overrightarrow{\mathrm{E}}_{\text {int }}=\sum_{k=1}^{n} \delta_{k} \vec{w}_{k} e_{k}, \\
& \overrightarrow{\mathrm{H}}_{\text {int }}=\sum_{k=1}^{n} \delta_{k} \vec{w}_{k} h_{k},
\end{aligned}
$$

where $\vec{w}_{k}$ is vector approximating function, $n$ is the number of edges on the element, $e_{k}$ and $h_{k}$ are unknown coefficients associated with each edge of the element. Vector approximating functions $\vec{w}_{k}$ are associated with $k^{t h}$ edge of the element by the following relation:

$$
\vec{w}_{k}=N_{i} \nabla N_{j}-N_{j} \nabla N_{i}
$$

where $N_{i}$ and $N_{j}$ are usual nodal approximating functions associated with endpoint nodes of $k^{\text {th }}$ element edge. With edge elements every global edge in the nonconformal tetrahedral mesh bust be given a global direction. This direction might differ from local direction of each edge and coefficient $\delta_{k}$ is either +1 or -1 depending on whether local edge direction coincides with global edge direction.

To apply FEM the Galerkin method is used. Galerkin method requires that the volume integral of vector dot product of $\delta_{k} \vec{w}_{k}$ and eqn (4) vanishes over the volume $V$ :

$$
\int_{V} \delta_{k} \vec{w}_{k} \cdot\left[\nabla \times\left(\frac{\mathrm{j}}{\omega \mu} \nabla \times \overrightarrow{\mathrm{E}}_{\text {int }}\right)-(\sigma+j \omega \epsilon) \overrightarrow{\mathrm{H}}_{\text {int }}\right] d V=0
$$

Using standard vector identity $\nabla \cdot \vec{P} \times \vec{Q}=\vec{Q} \cdot \nabla \times \vec{P}-\vec{P} \cdot \nabla \times \vec{Q}$ and divergence theorem the weak formulation is obtained:

$$
\int_{V} \frac{\mathrm{j}}{\omega \mu} \nabla \times \delta_{k} \vec{w}_{k} \cdot \nabla \times \overrightarrow{\mathrm{E}} d V+\int_{V}(\sigma+j \omega \epsilon) \delta_{k} \vec{w}_{k} \cdot \overrightarrow{\mathrm{E}} d V=\oint_{\partial V} d \vec{S} \cdot \delta_{k} \vec{w}_{k} \times \overrightarrow{\mathrm{H}}
$$

Note that notation $\overrightarrow{\mathrm{E}}_{\text {int }}$ and $\overrightarrow{\mathrm{H}}_{\text {int }}$ is dropped because of simplicity and that symbols $\overrightarrow{\mathrm{E}}$ and $\overrightarrow{\mathrm{H}}$ now denote the fields interior to volume $V$.

To convert exterior fields $\vec{E}_{\text {ext }}$ and $\vec{H}_{\text {ext }}$ in eqn (1) to interior fields $\overrightarrow{\mathrm{E}}$ and $\overrightarrow{\mathrm{H}}$ we make use of physical requirement that $\vec{n} \times \vec{H}_{\text {ext }}=\vec{n} \times \vec{H}, \vec{n} \times \vec{E}_{\text {ext }}=\vec{n} \times \vec{E}$. Furthermore, in order to treat boundary integral eqn (1) with FEM we take the cross product of whole eqn (1) with unit surface normal $\vec{n}$ (normal to surface $\partial V$ ) to obtain:

$$
\begin{gathered}
\alpha \vec{n} \times \vec{H}=\vec{n} \times \vec{H}_{i}+j \omega \epsilon \vec{n} \times \oint_{\partial V} d \vec{S}^{\prime} \times \vec{E} G+\vec{n} \times \oint_{\partial V}\left(d \vec{S}^{\prime} \times \vec{H}\right) \times \nabla^{\prime} G \\
-\frac{j}{\omega \mu} \vec{n} \times \oint_{\partial V} d S^{\prime} \nabla_{S}{ }^{\prime} \cdot\left(\vec{n}^{\prime} \times \vec{H}\right) \nabla^{\prime} G .
\end{gathered}
$$

Finally, to produce a linear system of equations, the eqn (10) is treated with Galerkin FEM by requiring that surface integral of dot product eqn (10) with interpolating functions $\delta_{k} \vec{w}_{k}$ vanishes: 


$$
\begin{aligned}
\int_{\partial V} d S \alpha \delta_{k} \vec{w}_{k} \cdot \vec{n} & \times \vec{H} \\
& =\oint_{\partial V} d S \delta_{k} \vec{w}_{k} \cdot \vec{n} \times \vec{H}_{i}+j \omega \epsilon \oint_{\partial V} d S \delta_{k} \vec{w}_{k} \cdot \oint_{\partial V} d \vec{S}^{\prime} \times \vec{E} G \\
& +\oint_{\partial V} d S \delta_{k} \vec{w}_{k} \cdot \vec{n} \times \oint_{\partial V}\left(d \vec{S}^{\prime} \times \vec{H}\right) \times \nabla^{\prime} G \\
& -\frac{j}{\omega \mu} \oint d S \delta_{k} \vec{w}_{k} \cdot \vec{n} \times \oint_{\partial V} d S^{\prime} \nabla_{S}{ }^{\prime} \cdot\left(\vec{n}^{\prime} \times \vec{H}\right) \nabla^{\prime} G .
\end{aligned}
$$

Using standard FEM procedures to eqns (9) and (11) one can now obtain a linear system of equations written in matrix form as:

$$
\left[\begin{array}{ccc}
H & G & 0 \\
D & M & M \\
0 & M & M
\end{array}\right]\left\{\begin{array}{l}
h_{b} \\
e_{b} \\
e_{v}
\end{array}\right\}=\left\{\begin{array}{c}
h_{I} \\
0 \\
0
\end{array}\right\}
$$

where $e_{I}$ are edge element coefficients computed from incident field $\overrightarrow{\mathrm{H}}_{\mathrm{i}}$ (using first right hand side term of eqn (11)), coefficients $h_{b}$ and $e_{b}$ are edge element coefficients associated with edges on computational boundary $\partial V$ and $e_{v}$ are edge element coefficients associated with edges that are inside computational domain $V$ ( and not on computational boundary $\partial V$ ). By solving the system of eqn (12) one can recreate electric field inside each tetrahedron in computational domain $V$ using eqn (5). However, for RCS computation we are only interested in coefficients $h_{b}$ and $e_{b}$ because these are associated with edges on computational boundary $\partial V$. Regarding the testing of this hybrid BEM/FEM computational method, the method was rigorously tested in various physical settings over the course of several years (see [12], [15], [16], [20]).

\section{COMPUTATION OF RADAR CROSS SECTION}

Once the edge element coefficients $h_{b}$ and $e_{b}$ are computed one can directly compute RCS from these coefficients without transforming near electromagnetic fields to far fields by using NTFFT by implementing technique developed by authors of this paper [14]. Radar cross scattering section $\sigma$ is defined as ratio of backscattered and incident field:

$$
\sigma=\lim _{|\vec{r}| \rightarrow \infty} 4 \pi|\vec{r}|^{2} \frac{\left|\vec{E}_{S}\right|^{2}}{\left|\vec{E}_{i}\right|^{2}}=\lim _{|\vec{r}| \rightarrow \infty} 4 \pi|\vec{r}|^{2} \frac{\vec{E}_{S} \cdot \vec{E}_{S}^{*}}{\vec{E}_{I} \cdot \vec{E}_{I}^{*}}
$$

where $\vec{E}_{S}^{*}$ represents the complex conjugate of backscattered vector field $\bar{E}_{S}$. In Ref. [14] it was shown that this backscattered field can be written as:

$$
\vec{E}_{S}=\frac{e^{-i k|\vec{r}|}}{4 \pi|\vec{r}|} \vec{F}_{S}
$$

where complex vector $\vec{F}_{S}$ can be computed from edge element coefficients as the sum: 


$$
\begin{aligned}
\vec{F}_{S}\left(\vec{e}_{\rho}\right)=-i \omega \mu \sum_{i=1}^{N} \sum_{j=1}^{3} \int_{\Delta_{i}} h_{b j} e^{i k \vec{r}, \cdot \vec{e}_{\rho}} d \vec{S}^{\prime} \times \delta_{j} \vec{w}_{j} \\
+i k \sum_{i=1}^{N} \sum_{j=1}^{3} \int_{\Delta_{i}} e_{b j} e^{i k \vec{r}^{\prime} \cdot \vec{e}_{\rho}}\left(d \vec{S}^{\prime} \times \delta_{j} \vec{w}_{j}\right) \times \vec{e}_{\rho} \\
\\
-i k \sum_{i=1}^{N} \sum_{j=1}^{3} \int_{\Delta_{i}} h_{b j} e^{i k \vec{r} \cdot \cdot \vec{e}_{\rho}} d S^{\prime \frac{\nabla^{\prime} S^{\prime} \cdot\left(\vec{n}^{\prime} \times \vec{w}_{j}\right)}{\sigma+i \omega \epsilon} \vec{e}_{\rho}}
\end{aligned}
$$

Computation of vector function $\vec{F}_{S}\left(\vec{e}_{\rho}\right)$ from known boundary edge coefficients $h_{b}$ and $e_{b}$ is fast because there are no numerical computations of double surface integrals as in eqn (11) and because there are no hyper-singular integrals involved in the computation.

The method is tested for a number of different configurations of inhomogeneous scattering problems and some previously published results [12] of the comparison of the method with results obtained by commercial software FEKO are shown in Fig. 2. FEKO software uses hybrid MoM/FEM to calculate near field and from there it computes RCS using near-to-far field transformation (NTFFT). As shown at the bottom of Fig. 2, the plane wave was directed along negative $\mathrm{z}$ axis at composite cone-sphere object. The first top layer of the cone is dielectric with relative permittivity $\epsilon_{r}=20$ and of height $0.2 \lambda$ (where $\lambda$ is the wavelength). Second layer of the cone is dielectric with relative permittivity $\epsilon_{r}=2$ and of height $0.3 \lambda$. The cone is placed on perfect electric conductor (PEC) hemisphere of radius $0.5 \lambda$. The arrangement is difficult for solvers because there is sharp metallic tip where some problems at corners are to be expected. However, as shown in Fig. 2, FEKO and hybrid $\mathrm{BEM} / \mathrm{FEM}$ described in this paper are in excellent agreement.

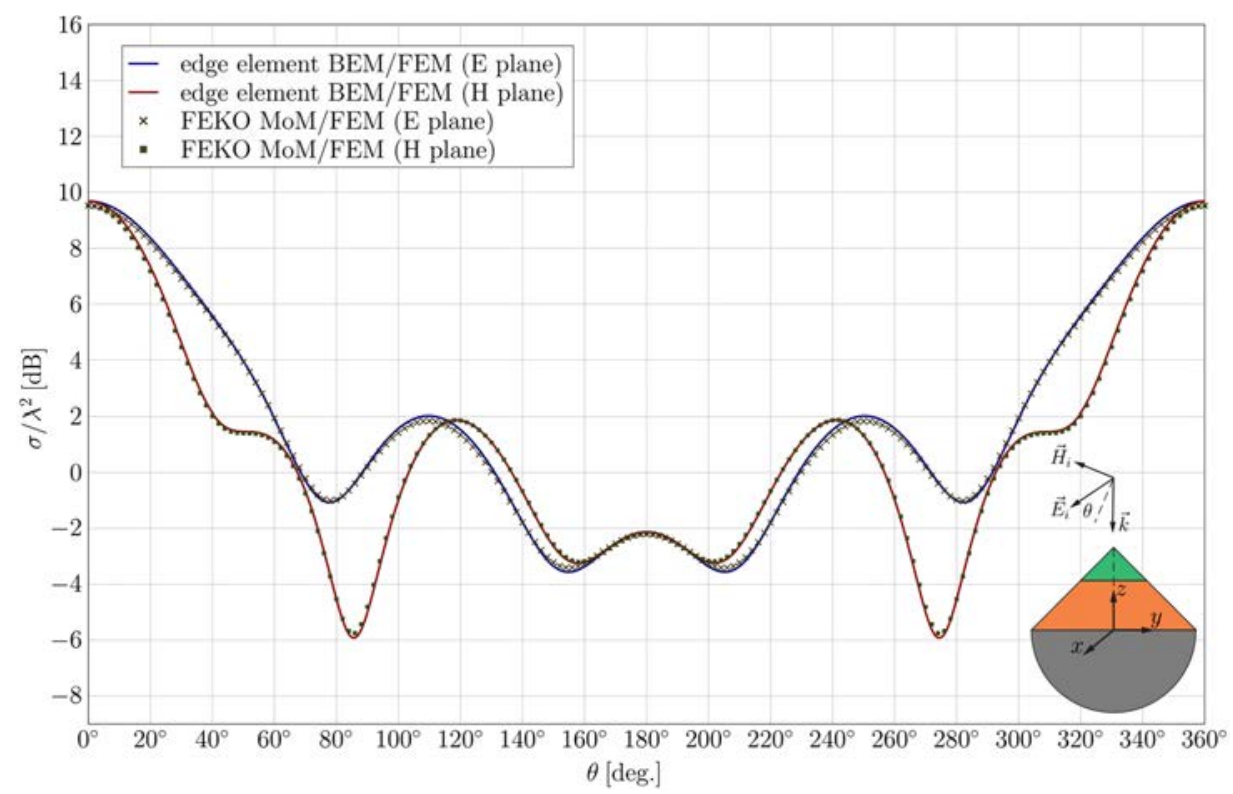

Figure 2: Bistatic RCS of composite three layered sphere-cone object computed by hybrid $\mathrm{BEM} / \mathrm{FEM}$ compared to results obtained by commercial software FEKO [11]. 


\section{MODELS OF SMALL MARITIME OBJECTS}

The rubber boat chosen for RCS computation is small service rubber boat used as a service boat in Croatian Navy. To correctly capture the geometry of the rubber boat it was subjected to series of 3D laser measurements as shown in Fig. 3. Laser measurement equipment was made available to authors by courtesy of Croatian Navy laser measurement facility site. Results of laser measurements were exported in the standard STL file format in order to produce nonconformal tetrahedral mesh of good quality the STL file was converted to Parasolid x_t CAD format using Geomagic software. The seawater shown in Fig. 4(a) into which the boat is submerged was added with Siemens SolidEdge CAD software. Nonconformal tetrahedral mesh shown in Fig. 4(b) was produced by Ansys ICEM software and it consists of 285,064 tetrahedral elements for FEM part of computation and of 3,632 triangular elements for BEM part of computation. Electrical parameters $\sigma, \epsilon_{r}, \mu_{r}$ of the seawater, air, rubber and plastic were compiled from various sources from literature [21] and these are given in Table 1.

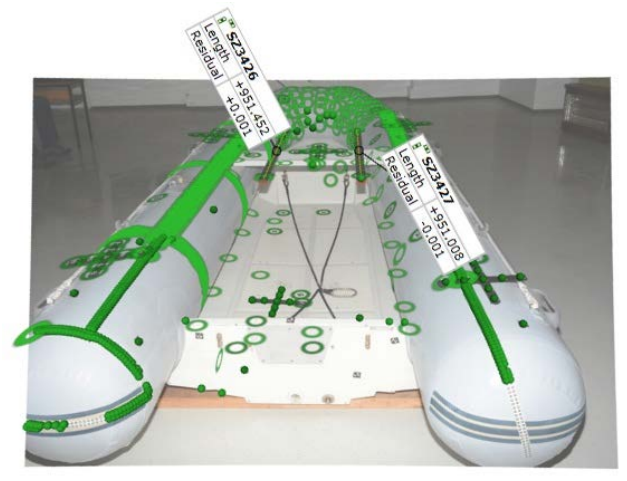

(a)

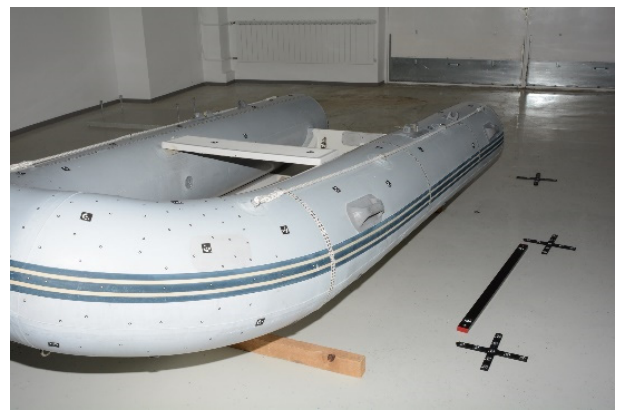

(b)

Figure 3: Photo of the rubber boat at laser measurement site is displayed in (a). The geometric features of the boat are captured at spatial points marked with green dots. In (b) the preparation of laser measurement site is shown.

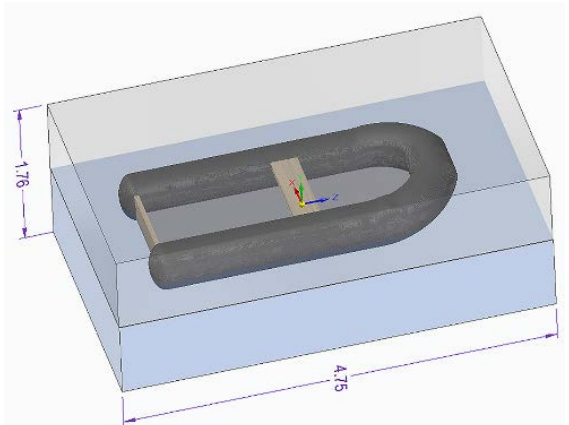

(a)

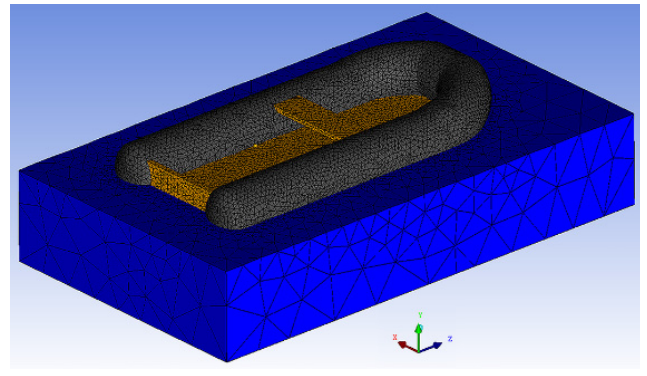

(b)

Figure 4: CAD model of the geometry for RCS computation is shown in (a). The rubber boat is partially immersed in seawater. In (b) nonconformal tetrahedral mesh of the rubber boat is displayed. 
Table 1: Electrical properties of materials for EM scattering calculation.

\begin{tabular}{|c|c|c|c|}
\hline \multirow{2}{*}{$\#$} & \multicolumn{3}{|c|}{ Electrical properties of materials } \\
\cline { 2 - 4 } & Material & $\boldsymbol{\sigma}[\mathrm{S} / \mathrm{m}]$ & $\boldsymbol{\epsilon}_{\boldsymbol{r}}$ \\
\hline 1. & Seawater & 3.00 & 88.00 \\
\hline 2. & Air & 0.00 & 1.00 \\
\hline 3. & Rubber & 0.00 & 2.50 \\
\hline 4. & Plastic & 3.00 & 3.00 \\
\hline 5. & Industrial steel & $6.21 \times 10^{6}$ & 1.00 \\
\hline
\end{tabular}

The CAD model of standard navigational buoy used along Croatian coastal area is depicted in Fig. 5(a). The model was built from the data made available by Croatian Hydrographic Institute (HHI). Nonconformal tetrahedral mesh of the buoy model and of surrounding seawater consisted of 986,260 tetrahedral elements and 1,870 elements for BEM surface.

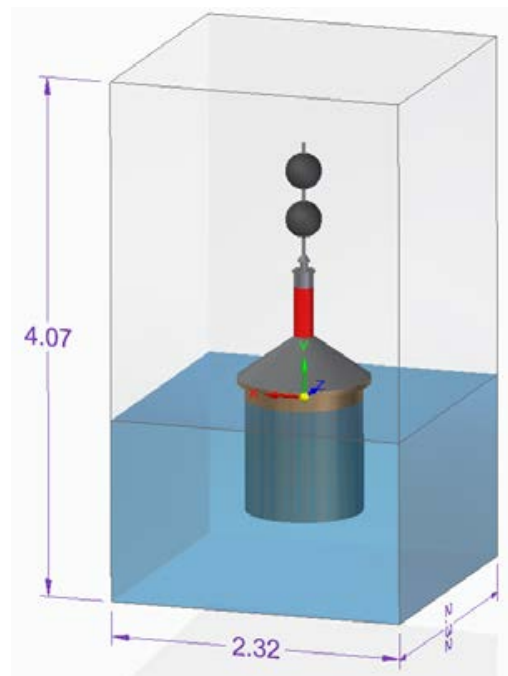

(a)

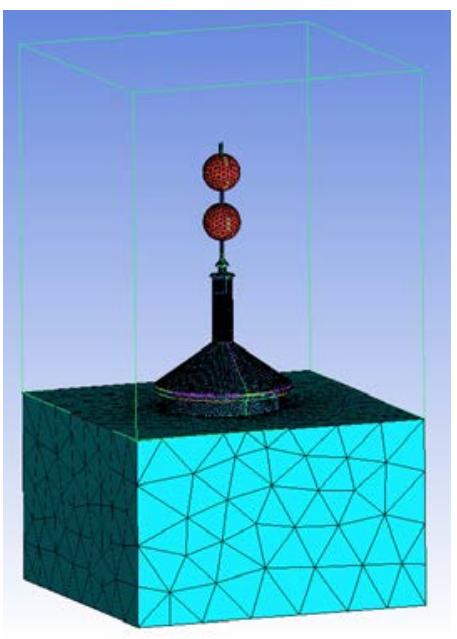

(b)

Figure 5: CAD model of the navigational buoy partially submerged into seawater is displayed in (a). Nonconformal tetrahedral mesh of the buoy and seawater is shown in (b). Model consists of 986,260 tetrahedral elements and 1,870 triangular elements to model the computational boundary $\partial \mathrm{V}$.

\section{NUMERICAL RCS RESULTS}

Physical setting for RCS computation of rubber boat and the buoy is displayed in Fig. 6. The rubber boat is at distance $l$ from the ship and the radar antenna is located at height $h$ above the sea level. In Fig. 6 angle $\theta$ represents azimuth, that is, the angle $\theta$ is the angle between the imaginary line connecting radar antenna and the rubber boat (buoy). Furthermore, RCS computations were performed for both horizontal polarization (HP) and vertical polarization 
(VP). The direction of EM wave propagation is always along imaginary line connecting radar antenna and the rubber boat. For HP electric field is in the sea plane and for VP the magnetic field is in the sea plane.

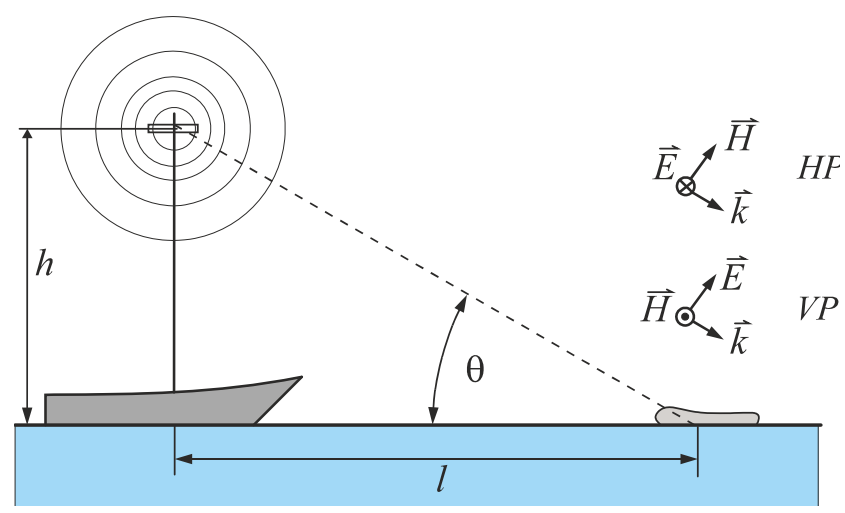

Figure 6: The rubber boat is at horizontal distance $l$ from the radar antenna and the radar antenna is at height $h$ above sea level. Angle subtended between the line connecting the antenna and boat and between sea level is $\theta$.

Normally, electromagnetic waves reflect from the sea surface and even the sea itself has radar cross section. In order for radar to distinguish between the target (rubber boat, buoy) and the sea, the RCS has to be different. In fact, we could devise a measure corresponding to the ratio of RCS of the sea itself and the target which could indicate how much the target distinguishes itself from the sea. Because of this we have first calculated the radar cross section $\sigma_{S}$ of the sea itself as a function of various azimuth angles $\theta$, and the results for both HP and VP are shown in Fig. 7(a).

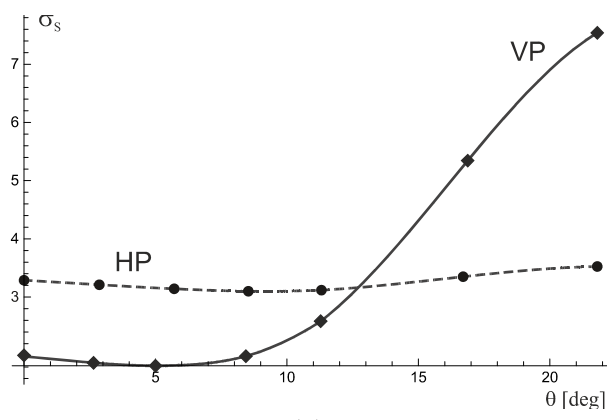

(a)

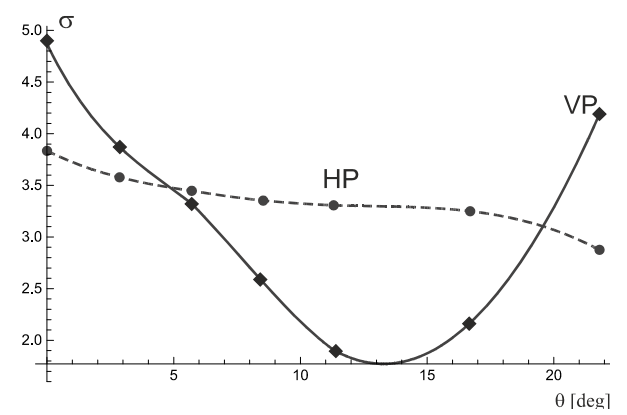

(b)

Figure 7: Radar cross section of the seawater (without rubber boat) for various incidence angles $\theta$ is shown in (a) for horizontal polarization (HP) and vertical polarization (VP). Radar cross section of both rubber boat and seawater is shown in (b) for HP and VP. 
The calculations of radar cross section $\sigma$ of rubber boat immersed in the sea, depicted in Fig. 4(b), is carried out next. Various azimuth angles are considered and the results for both HP and VP are displayed in Fig. 7(b). In order to find out how much radar cross section of the rubber boat distinguishes itself from the radar cross section of the sea the ratio $\sigma / \sigma_{S}$ was calculated as shown in Fig. 8. When ratio $\sigma / \sigma_{S}$ is close to 1 it is impossible for radar to distinguish the rubber boat from sea surface.

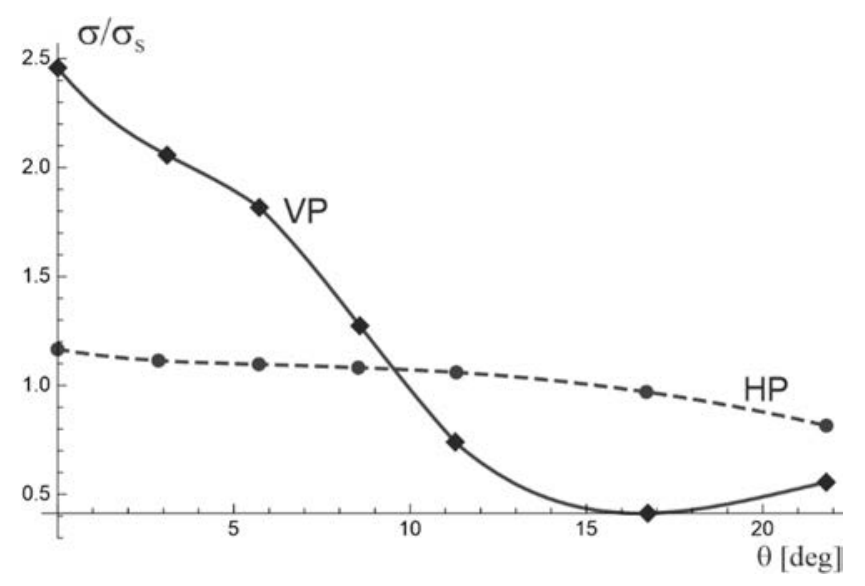

Figure 8: The ratio $\sigma / \sigma_{S}$ for rubber boat for both HP and VP polarization.

Similar calculations of the $\sigma / \sigma_{S}$ ratio are performed for buoy for both HP and VP polarization and the results of this calculations are shown in Fig. 9.

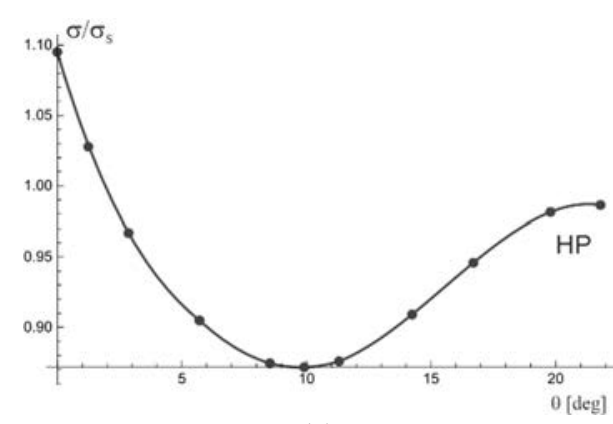

(a)

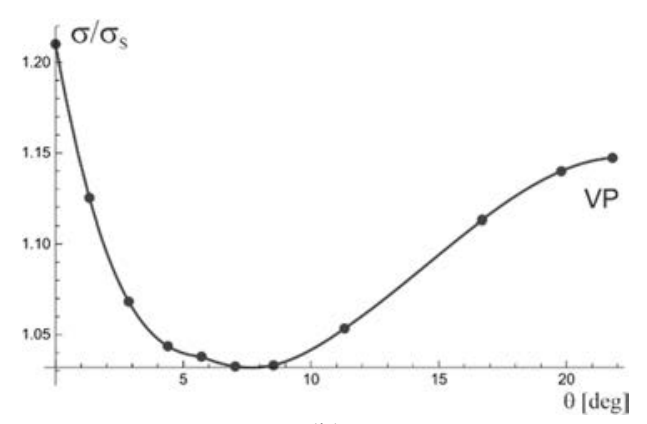

(b)

Figure 9: The ratio of cross section $\sigma / \sigma_{S}$ for buoy is shown for horizontal polarization in (a). In (b) the ratio $\sigma / \sigma_{S}$ for buoy is shown for vertical polarization (VP).

The calculations of near electromagnetic fields for rubber boat and the buoy are shown in Fig. 10. From these field values RCS was calculated using eqn (15). The results shown in Figs $8-10$ indicate that there are some azimuth angles $\theta$ where it is very difficult for radar to distinguish small target from the surface. Hence, further research will be carried out to investigate this possible safety issue. 


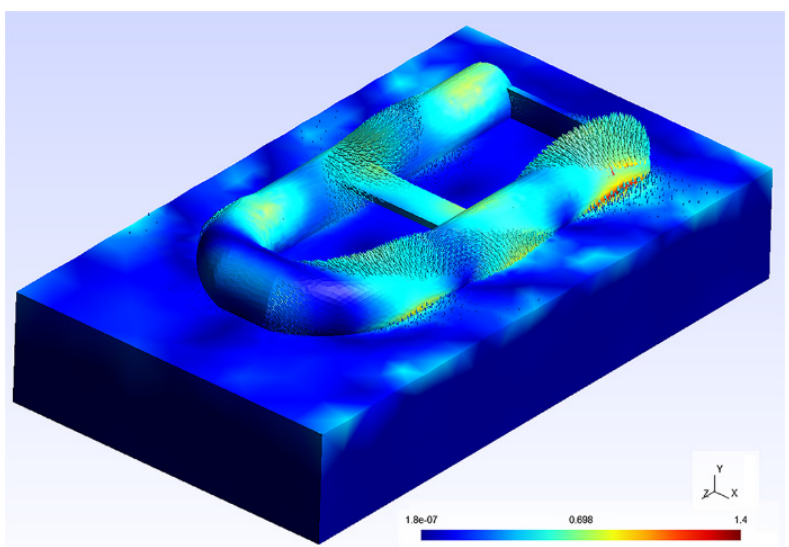

(a)

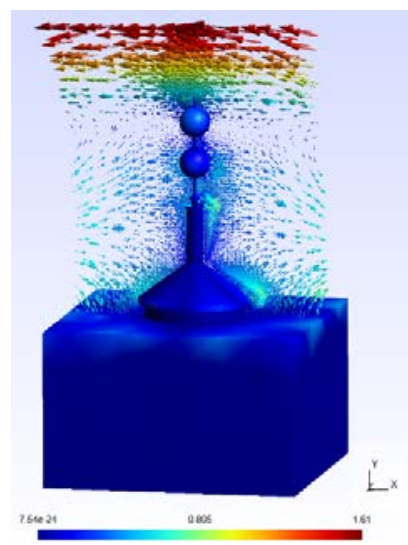

(b)

Figure 10: The vectors of near electric field are shown on the surface of rubber boat in (a), and the vectors of near electric field are shown in the air surrounding the navigational buoy in (b).

\section{CONCLUSION}

The present paper outlines an efficient method for RCS calculation of small maritime radar targets. The method presented is full-wave method that can take into account the material inhomogeneities of radar targets. The near field computation was performed using hybrid $\mathrm{BEM} / \mathrm{FEM}$ with edge elements which bear certain advantages over RWG elements that are standardly used with competing MoM/FEM formulation: these elements are divergence-less (divergence free), hence, they naturally satisfy zero divergence condition and they preserve tangential continuity of electromagnetic fields across inter-element boundaries which is physically required condition. From near field values the RCS was computed using formulation based on edge elements. Namely, the results for RCS as a function of azimuth, are given for small maritime objects, i.e. small rubber boat and buoy. The RCS values for these small objects are compared to RCS of the seawater and some conclusions regarding detectability of small maritime objects by radar were drawn.

\section{ACKNOWLEDGEMENT}

We thank Mr. Ljubomir Ostavić from Croatian Ministry of Defense for performing the laser measurements of the service rubber boat.

\section{REFERENCES}

[1] Maini, A.K., Handbook of Defence Electronics and Optronics: Fundamentals, Technologies and Systems, John Wiley \& Sons Ltd.: New York, pp. 113-202, 2018.

[2] Bole, A. et al., Radar and ARPA Manual, Elsevier: London, pp. 139-213.

[3] Knott, E.F., A progression of high-frequency RCS prediction techniques. Proceedings of the IEEE, 73(2), pp. 252-264, 1985.

[4] Youssef, N.N., Radar cross section of complex targets. Proceedings of the IEEE, 77(5), pp. 722-734, 1989.

[5] Silverstein, J.D. \& Bender, R., Measurements and predictions of the RCS of Bruderhedrals at millimeter wavelengths. IEEE Transactions on Antennas and Propagation, 45(7), pp. 1071-1079, 1997. 
[6] Weinmann, F., Ray tracing with PO/PTD for RCS modeling of large complex objects. IEEE Transactions on Antennas and Propagation, 54(6), p. 1806, 2006.

[7] Dodig, H. et al., Edge element calculation of radar cross section of small maritime targets with respect to height of radar antenna. TransNav: International Journal on Marine Navigation and Safety of Sea Transportation, 14(2), pp. 331-336, 2020.

[8] Liu, J. et al., An efficient ray-tracing method for RCS prediction in GRECO. Microwave and Optical Technology Letters, 55, pp. 586-589, 2012.

[9] Yee, K., Numerical solution of initial boundary value problems involving Maxwell's equations in isotropic media. IEEE Transactions on Antennas and Propagation, 14(3), pp. 302-307, 1966.

[10] $\mathrm{Xu}, \mathrm{R} . \mathrm{W}$. et al., A hybrid FEM/MoM technique for 3-D electromagnetic scattering from a dielectric object above a conductive rough surface. IEEE Geoscience and Remote Sensing Letters, 13(3), pp. 314-318, 2016.

[11] Dodig, H. et al., On the computation of singular integrals over triangular surfaces in R3. Boundary Elements and Other Mesh Reduction Methods XLI, eds A. Cheng \& S. Syngellakis, WIT Press: Southampton, UK, pp. 95-105, 2018.

[12] Dodig, H. et al., On the edge element boundary element method/finite element method coupling for time harmonic electromagnetic scattering problems. International Journal for Numerical Methods in Engineering, early view (online), pp. 1-40, 2021.

[13] Taflove, A. \& Hagness, S.C., Computational Electrodynamics: The Finite-Difference Time-Domain Method, Artech House Inc.: Boston, pp. 329-352, 2005.

[14] Dodig, H., A boundary integral method for numerical computation of radar cross section of 3D targets using hybrid BEM/FEM with edge elements. Journal of Computational Physics, 348, pp. 790-802, 2017.

[15] Dodig, H. et al., Hybrid BEM/FEM edge element computation of the thermal rise in the 3D model of the human eye induced by high frequency EM waves. Proceedings of SOFTCOM, 2012.

[16] Dodig, H. et al., Stochastic sensitivity of the electromagnetic distributions inside a human eye modeled with a 3D hybrid BEM/FEM edge element method. Engineering Analysis with Boundary Elements, 49, pp. 48-62, 2014.

[17] Stratton, J. \& Chu, L.J., Diffraction theory of electromagnetic waves. Physical Review, 56, pp. 99-107, 1939.

[18] Harrington, R.F., Time-Harmonic Electromagnetic Fields, John Wiley \& Sons: New York, p. 77, 2001.

[19] Nedelec, J.C., Mixed finite elements in R3. Numerische Mathematik, 35, pp. 315-341, 1980.

[20] Cvetković, M. et al., A study on the use of compound and extracted models in the high frequency electromagnetic exposure assessment. Mathematical Problems in Engineering, web only/open access, 2017, 7932604, 2017.

[21] Garazza, A.R.L. et al., Influence of the microstructure of vulcanized polybutadiene rubber on the dielectric properties. Polymer Testing, 30, pp. 657-662, 2011. 